\title{
Short communication: Use of an ultrafiltration device in gland cistern for continuous sampling of healthy and mastitic quarters of lactating cattle for pharmacokinetic modeling
}

\author{
D. A. Mzyk, ${ }^{*}$ C. M. Bublitz, ${ }^{*}$ H. Sylvester, ${ }^{*}$ K. A. E. Mullen,† G. D. Hobgood, ${ }^{*}$ R. E. Baynes, ${ }^{*}$ and D. M. Foster ${ }^{* 1}$ \\ *Department of Population Health and Pathobiology and the Food Animal Residue Avoidance Depletion Program, College of Veterinary Medicine, \\ and
}

†Department of Animal Science, North Carolina State University, Raleigh 27695

\begin{abstract}
Pharmacokinetic studies of the drugs in the milk are often limited due to infrequent sampling associated with milking. Alternatively, frequent sample collection with repeated milking may increase drug elimination. The objective of this study was to determine the feasibility of continuously sampling the udder using ultrafiltration. An ultrafiltration probe was placed into the gland cisterns through mammary parenchyma of normal and mastitic quarters of 6 mature mid-lactation Jersey cows with naturally occurring subclinical mastitis. An ultrafiltration probe was secured to the caudal or lateral aspect of the udder depending on the quarter being sampled. The timed interval samples were collected at $0,2,4,6,8,12,18,24,28,32,36,48,60,72,84$, and $96 \mathrm{~h}$ after drug administration. Plasma samples were collected at the same time points. Each cow received $2.2 \mathrm{mg} / \mathrm{kg}$ of flunixin intravenously before milking at time 0 . All cows were routinely milked by machine every $12 \mathrm{~h}$. Flunixin concentrations in plasma, whole milk, and milk ultrafiltrates were analyzed by use of ultra-high-performance liquid chromatography with mass spectrometric detection. We found no significant effects on the appearance of the milk or the ability to milk the cows after implantation of the ultrafiltration probes. The concentration of flunixin collected from the ultrafiltration probes in the mastitic quarters tended to be greater than that of the healthy quarters. We concluded that collection of ultrafiltration samples from the mammary gland of cows provides a viable means to continuously assess drug concentrations in the milk while continuing to milk the cow normally. This study demonstrates the utility of continuous sampling of milk via ultrafiltration for future pharmacokinetic studies in cattle.
\end{abstract}

Received March 30, 2018.

Accepted July 16, 2018.

${ }^{1}$ Corresponding author: dmfoster@ncsu.edu
Key words: ultrafiltration, pharmacokinetic, dairy cattle, gland cistern

\section{Short Communication}

The treatment of mastitis accounts for a large proportion of therapeutic drug use on dairy farms. Nonsteroidal anti-inflammatory drugs such as flunixin are reportedly the second most prescribed class of drugs by dairy veterinarians (Sundlof et al., 1995), surpassed only by intramammary antibiotics. Flunixin meglumine is a nonsteroidal anti-inflammatory approved for use in beef and dairy cattle for the modulation of inflammation in endotoxemia and the control of pyrexia associated with bovine respiratory disease and acute mastitis. Mastitis can alter drug distribution and clearance, and whole milk samples may not adequately represent the drug concentration-time profiles. In many pharmacokinetic (PK) studies, investigators measure total drug concentrations in milk samples, either from the individually treated quarters or the bucket milk (Cagnardi et al., 2014; Stockler et al., 2009). Milk fraction sampled is often not specified. Previous studies have reported differences in sample collection technique during milking procedures for antibiotic quantification. This includes foremilk, total milk, or postmilk samples during the milking process (Stockler et al., 2009). These differences may be due to concentrations of the drug not distributing evenly in the mammary gland or milk fractions varying in protein and fat percentages as well as drug concentration. Further, drugs have been shown to have different percentages of binding to the proteins found in milk, including lactoferrin, albumin, and casein, which could greatly influence drug distribution and PK analysis (Stebler and Guentert, 1990). Another limitation of PK studies evaluating milk concentrations of drugs is that samples can either be collected at the time of milking (2-3 times a day), which may be too infrequent for accurate PK modeling, or through more frequent collection of milk, which may artificially increase the 
clearance of the drug. Continuous sampling without removal of large volumes of milk would be ideal.

The use of UF probes have been used successfully to collect interstitial fluid of many different species, including dogs (Bidgood and Papich, 2003; Davis et al., 2006, 2007; Messenger et al., 2012), horses (Bidgood and Papich, 2003; Davis et al., 2006, 2007; Messenger et al., 2012; Underwood et al., 2014), pigs (Bidgood and Papich, 2003; Davis et al., 2006, 2007; Messenger et al., 2012), calves (Warren et al., 2014), and sheep (Sojka et al., 2000). These probes have typically been placed in the subcutaneous space, but other locations, including pleural space (Davis et al., 2007), bone or muscle (Sojka et al., 2000), gastrointestinal tract (Warren et al., 2014) and lamellar tissue (Underwood et al., 2014), have also been examined.

The UF probe contains 3 semipermeable loops connected to a tube extending to the exterior of the animal and attached to a Vacutainer tube (BD Vacutainer, BD, Franklin Lakes, NJ). This tube, which provides negative pressure for fluid collection through small pores in the loop membrane, allows water, electrolytes, and low-molecular weight molecules (less than 30,000 Da) to pass. Larger molecules, including proteins, proteinbound drugs, and fats, are excluded. To determine appropriate treatment regimens to maximize therapeutic success, accurate PK data utilizing unbound (free) drug concentration is needed at different sites of action. For infections in the mammary gland, free drug concentrations in the gland cistern are related to therapeutic efficacy. Therefore, the objective of our study was to evaluate the use of a UF device placed in the gland cistern in lactating dairy cattle as a new continuous sampling technique to determine active drug concentrations in the mammary gland.

This study was approved by the Institutional Animal Care and Use Committee at North Carolina State University. Six lactating Jersey dairy cows, weighing from 294 to $602 \mathrm{~kg}$ and from 72 to $152 \mathrm{~d}$ in lactation were used. Cows were housed in individual stalls bedded with shavings and were fed a concentrate mix and ad libitum alfalfa hay with free access to water for the duration of the study. All quarters of each cow were cultured to assess the health of the quarter before probe insertion. Each cow was selected for inclusion in the study if at least 1 quarter had no microbial growth from milk culture. Two cows with naturally occurring, subclinical Staphylococcus aureus infection in at least 1 quarter and 3 cows with CNS infections were enrolled in the study. One cow enrolled had no microbial growth from milk culture. A total of 3 healthy quarters and 5 mastitic quarters were included in the study. These infections had been present for an average of $10 \mathrm{~d}$ at the time of the study. Cows were milked every $12 \mathrm{~h}$ throughout the study using a portable milking machine (GEA Group, Dusseldorf, Germany).

At time 0 , each cow was administered $2.2 \mathrm{mg} / \mathrm{kg}$ of flunixin meglumine (Flunixamine Injectable Solution, Zoetis Inc., Parsippany-Troy Hills, NJ) intravenously. Flunixin meglumine was administered immediately after milking.

Prior to drug administration, a 14-gauge, $13.3-\mathrm{cm}$ jugular catheter (BD Angiocath, Becton Dickinson, Infusion Therapy Systems Inc., Sandy, UT) was inserted in the jugular vein for the purpose of collecting blood samples. Whole blood samples were collected into heparinized tubes (BD Vacutainer) before dosing and at appropriate intervals for optimum PK modeling, accounting for $90 \%$ of drug elimination. Blood was collected at 0,15 , and $30 \mathrm{~min}$ and $1,2,4,6,8,12,24,26,28,30$, $32,36,48,60,72,84$, and $96 \mathrm{~h}$ after flunixin meglumine administration. Whole blood was centrifuged at 1,700 $\times g(3,500 \mathrm{rpm})$ for $10 \mathrm{~min}$ at room temperature, and the resulting plasma was transferred into cryogenic vials and immediately frozen at $-80^{\circ} \mathrm{C}$ until analysis.

Ultrafiltrate fluid collection was performed with a reinforced in vivo UF sampling kit (RUF-3-12, BAS; Bioanalytical Systems, West Lafayette, IN). All 4 cows were sedated with an intramuscular injection of 0.025 $\mathrm{mg} / \mathrm{kg}$ of butorphanol (Butorphic, $10 \mathrm{mg} / \mathrm{mL}$; Akorn Inc., Lake Forest, IL), $0.1 \mathrm{mg} / \mathrm{kg}$ of ketamine (KetaVed, $100 \mathrm{mg} / \mathrm{mL}$; Vedco Inc., St. Joseph, MI), and $0.01 \mathrm{mg} /$ $\mathrm{kg}$ of xylazine (Rompun, $20 \mathrm{mg} / \mathrm{mL}$; Bayer Animal Health, Leverkusen, Germany). The gland cistern of the quarter being sampled (healthy: $\mathrm{n}=3$; mastitic: $\mathrm{n}=5$ ) was located using ultrasound (BCF Technology, Rochester, MN). After the skin was clipped and sterilized, $2 \mathrm{~mL}$ of lidocaine (Lidocaine HCL $20 \mathrm{mg} / \mathrm{mL}$ Injection, Vedco Inc.) was injected at the location of probe insertion for local anesthesia. A stab incision was made using a \#10 scalpel blade. A sterile 3.8-cm, 10-gauge needle was fitted with a Christmas tree adaptor then advanced under ultrasound guidance until the tip of the needle was inserted into the cistern (Figure 1). The adaptor was then removed to allow milk to drain from the quarter. After milk was drained from the cistern so no milk was flowing through the needle, the collecting end of the UF probe was inserted through the needle into the cistern so the entire collecting apparatus was within the lumen. The needle was then removed from the udder and a 2-0 nylon suture (Ethicon, Somerville, NJ) was placed to secure the probe. Tubing exiting the udder was secured on the lateral (front quarters) or caudal aspect (rear quarters) of the udder.

The tubing was connected to a needle within a vacuum vial needle holder using a small piece of flexible tubing of slightly larger diameter and secured with tape. The needle holder was sutured to the skin using 


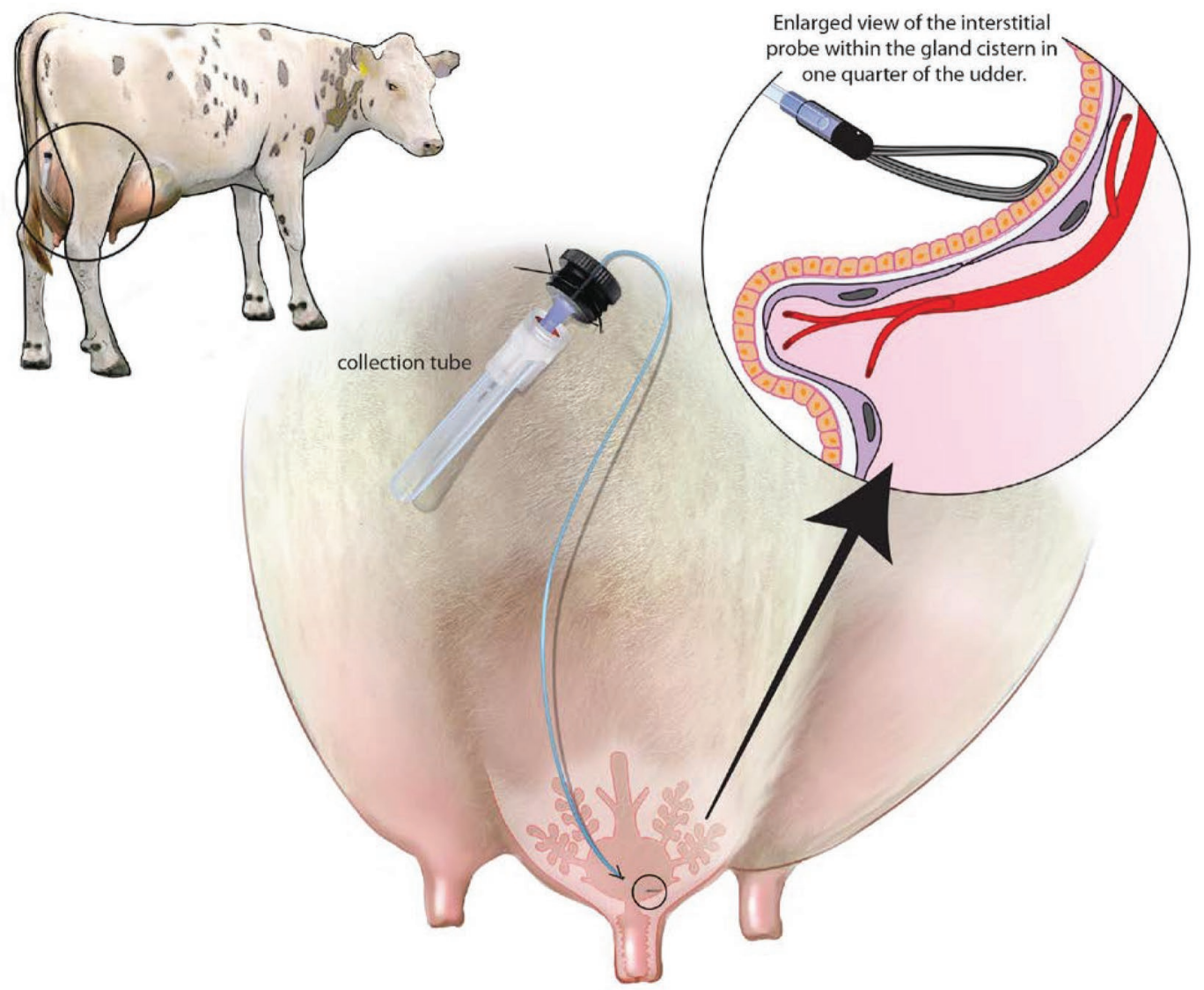

Figure 1. Medical illustration of probe placement in cistern. Color version available online.

2-0 nylon suture (Ethicon; Figure 2). To collect ultrafiltrate from the cistern, a $3-\mathrm{mL}$ evacuated tube with no additive (BD) was inserted onto the needle of the vacuum vial needle holder. The timed interval samples were collected at $0,2,4,6,8,12,18,24,28,32,36,48$, $60,72,84$, and $96 \mathrm{~h}$ after drug administration by changing the tubes at the predetermined time points. Whole milk samples were collected from individual quarters before milking (foremilk) every $12 \mathrm{~h}$ until $96 \mathrm{~h}$.

Flunixin (FLU) concentrations were quantified in all plasma, whole milk, and milk ultrafiltrate samples by use of ultra-high-performance liquid chromatography with mass spectrometric detection. An Acquity UPLC BEH C18 column $(1.7 \mu \mathrm{m}$ inside diameter, $2.1 \times 50$ mm; Waters, Milford, MA) with a guard column was used. Plasma and milk ultrafiltrate samples were extracted using a protein precipitation method. Briefly, $150 \mu \mathrm{L}$ of sample was combined with $750 \mu \mathrm{L}$ of $0.5 \%$ citric acid in acetonitrile. Samples were mixed with a vortex for $30 \mathrm{~s}$ and centrifuged for $10 \mathrm{~min}$ at $4,500 \times$ $g$ at room temperature. Plasma and milk ultrafiltrate supernatant was collected after centrifugation and placed into a clean $16 \times 125 \mathrm{~mm}$ glass culture tube
As an additional clean-up step for whole milk samples, the supernatant was loaded on a Supelco Hybrid SPEphospholipid cartridge (Sigma-Aldrich, St. Louis, MO). The eluate from the cartridge was collected and transferred to a $16 \times 125 \mathrm{~mm}$ glass culture tube. All glass culture tubes with supernatant were dried using rapid vacuum evaporation at $55^{\circ} \mathrm{C}$ for $15 \mathrm{~min}$ at $138 \mathrm{kPa}$ for evaporation to dryness. Samples were reconstituted in $300 \mu \mathrm{L}$ of 50:50 acetonitrile:water. The injection volume on the ultra-high-performance liquid chromatographyMS-MS was $5 \mu \mathrm{L}$ and concentrations were derived by comparing peak areas of samples with those from an external standard curve. The limits of detection and quantification for flunixin in plasma were 0.01 and 0.02 $\mu \mathrm{g} / \mathrm{mL}$, respectively, and the linear range was 0.02 to $30 \mu \mathrm{g} / \mathrm{mL}$. The limits of detection and quantification for flunixin in milk/milk ultrafiltrate were 0.001 and $0.005 \mu \mathrm{g} / \mathrm{mL}$, respectively, and the linear range for milk was 0.005 to $1 \mu \mathrm{g} / \mathrm{mL}$. Relative standard deviation for both interday and intraday accuracy was $<15 \%$ at all concentrations $\left(\mathrm{R}^{2}>0.99\right)$.

A noncompartmental analysis of FLU plasma concentration versus time profiles was performed with Phoenix 
pharmacokinetic modeling software (Pharsight Corp., St Louis, MO). The area under the plasma concentration time curve from time zero to infinity $\left(\mathbf{A} \mathbf{U} \mathbf{C}_{0 \rightarrow \infty}\right)$ was calculated by the linear trapezoidal rule. The rate constant $\left(\lambda_{z}\right)$, associated with the terminal elimination phase, was estimated by means of linear regression of the terminal phase of the log concentration versus time profile and the corresponding terminal half-life $\left(\mathrm{t}_{1 / 2 \lambda z}\right)$ were calculated. The rate constant also was used to extrapolate $\mathrm{AUC}_{0 \rightarrow \infty}$ from the time of the last observed concentration to infinity. The volume of distribution at steady state $\left(\mathrm{Vd}_{\mathrm{ss}}\right)$ was calculated. The $\mathrm{AUC}_{0 \rightarrow \infty}$ were used to calculate clearance (CL) and mean residence time (MRT).

When determining the ideal placement of the UF probes, there were 2 cows (not included in this study) had significant bleeding into the gland cistern. Due to this, we changed methods by using a shorter introducer needle and implemented the use of ultrasound to better visualize the cistern. To avoid excessive hemorrhage and minimize tissue trauma, probes were inserted at the base of the gland cistern. Once these improvements were made, we observed no significant effects on the appearance of the milk (in color or viscosity) or the ability to milk the cows after implantation of the UF probes. With sedation, the cows tolerated the implantation well and were not visibly bothered by the probes during sample collection. We were able to collect 127 out of 128 samples (representing 16 sampling time points for 8 different quarters with probes) for the entire study. At the end of the study, holding sutures were cut and the probes were removed without any issues.

Table 1 presents the mean plasma noncompartmental PK parameters following intravenous dosing. The mean FLU plasma concentrations decreased from 4.4 to $\leq 0.05 \mu \mathrm{g} / \mathrm{mL}$ by $24 \mathrm{~h}$ after dosing (Figure 3 ). The mean observed peak plasma concentration was $4.04 \pm$ $0.30 \mu \mathrm{g} / \mathrm{mL}$. The FLU was at or below the level of detection $(0.001 \mu \mathrm{g} / \mathrm{mL})$ in the plasma of all of the cows $48 \mathrm{~h}$ after intravenous administration.

Table 1. Flunixin plasma pharmacokinetic parameters after $2.2 \mathrm{mg} /$ $\mathrm{kg}$ of intravenous dosing $(\mathrm{n}=5)$

\begin{tabular}{llc}
\hline Parameter $^{1}$ & & Mean $\pm \mathrm{SD}$ \\
\hline $\mathrm{t}_{1 / 2 \lambda \mathrm{z}}$ & $\mathrm{h}$ & $3.38 \pm 1.33$ \\
$\lambda \mathrm{z}$ & $\mathrm{h}^{-1}$ & $0.25 \pm 0.16$ \\
$\mathrm{AUC}$ & $\mathrm{\mu g} \cdot \mathrm{h} / \mathrm{mL}$ & $8.80 \pm 3.2$ \\
$\mathrm{CL}$ & $\mathrm{mL} / \mathrm{hr}$ per kg & $276.6 \pm 98.3$ \\
$\mathrm{Vd}_{\mathrm{ss}}$ & $\mathrm{L} / \mathrm{kg}$ & $1,149.1 \pm 266.7$ \\
$\mathrm{MRT}$ & $\mathrm{hr}$ & $4.3 \pm 0.7$ \\
\hline
\end{tabular}

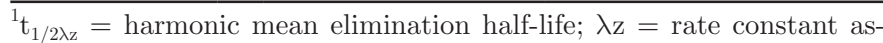
sociated with the terminal elimination phase; $\mathrm{AUC}_{0 \rightarrow \infty}=$ total area under the curve; $\mathrm{CL}=$ clearance; $\mathrm{Vd}_{\mathrm{ss}}=$ volume of distribution at steady state; $\mathrm{MRT}=$ mean residence time.
The concentrations determined in the milk ultrafiltrate are represented in Figure 4. Unlike plasma and total milk samples, 2 peak concentrations of FLU were identified, first around $12 \mathrm{~h}$ after dosing, followed by a second peak around $32 \mathrm{~h}$. This pattern was observed in both healthy and mastitic quarters. Flunixin concentrations in whole milk are depicted in Figure 5 and represent 3 healthy quarters and 5 quarters affected by mastitis. Only 1 peak concentration in whole milk samples from both healthy and mastitis quarters occurred at $12 \mathrm{~h}$ after dosing.

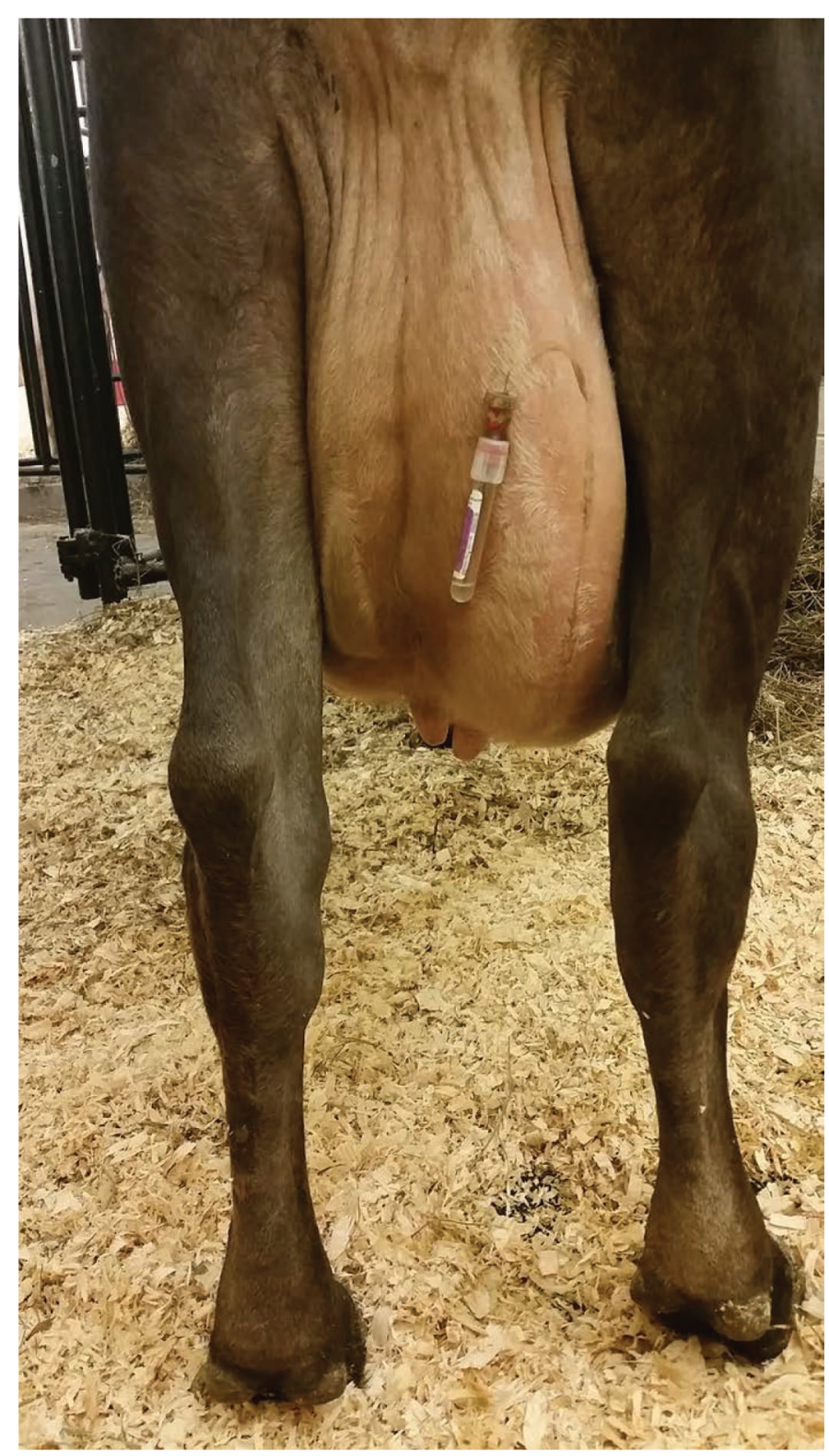

Figure 2. Picture of probe and tube placement. Color version available online. 
10418

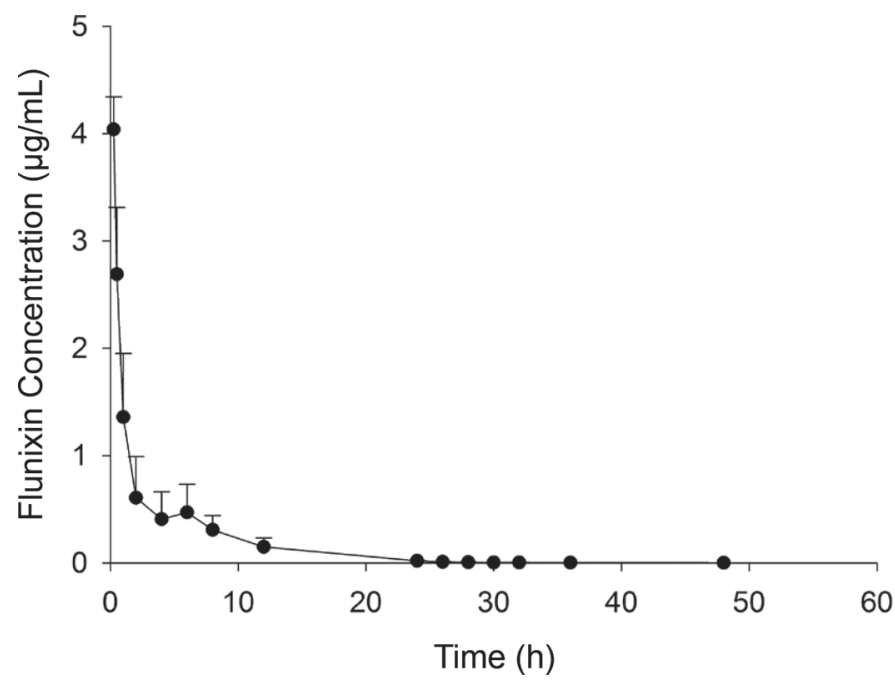

Figure 3. Total flunixin (mean $\pm \mathrm{SD}$ ) concentrations in plasma over $60 \mathrm{~h}$.

The objective of the present pilot study was to develop a novel sampling technique for use in the bovine mammary gland to better understand the partitioning of FLU into milk. The lack of data that accurately depict PK and pharmacodynamics within the bovine udder are largely due to the complex nature of the mammary gland and inability to quantify drug concentrations at the effector site (Stockler et al., 2009; Lainesse et al., 2012). Several study designs have attempted to improve the prediction of distribution or elimination for drugs used to treat mastitis, including an ex vivo bovine perfused udders, blood concentrations, and cell culture (Ehinger and Kietzmann, 2000; Al-Bataineh et al., 2009). The use of UF probes in the gland cistern

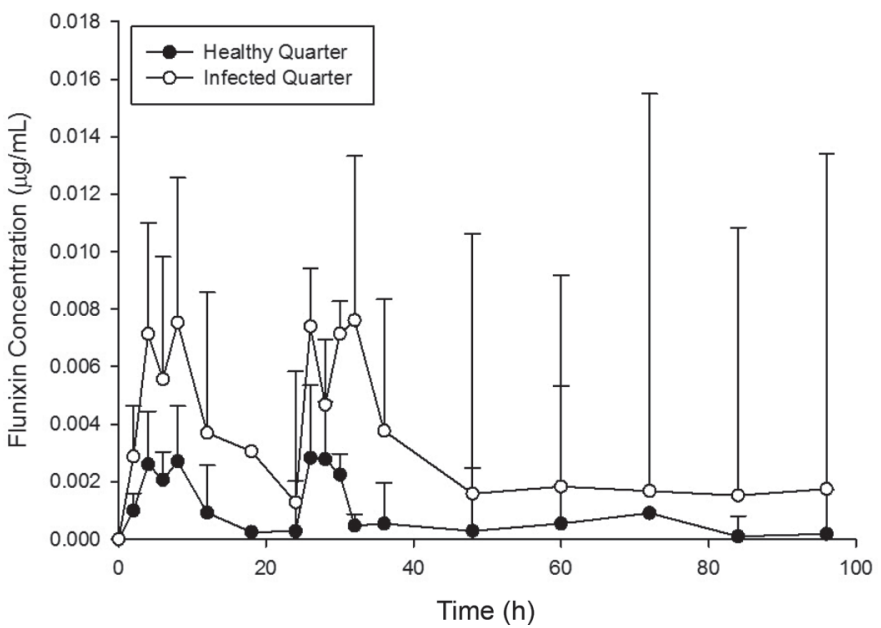

Figure 4. Geometric mean \pm SD flunixin concentrations using UF probes in healthy and mastitic quarters following single-dose administration of intravenous flunixin meglumine. described in the current study allows for minimally invasive and continuous sampling of milk within the gland cistern. In addition, the influence of pathophysiological differences between the healthy and mastitic udders can be assessed within the same animal. This technique was improved by use ultrasound guidance to determine depth of the gland cistern and helped avoid major vessels, as initial attempts led to significant bleeding within the cistern. Securely suturing the probe into place is critical, as the suction created by milking procedure can pull the probe through the streak canal.

Therapeutic agents that are administered intramuscularly are formulated to produce a local therapeutic effect without any need for significant systemic drug exposure. In those cases, quantifying blood levels of drugs are inadequate to accurately describe drug concentrations in gland cistern. To determine appropriate treatment regimens to maximize therapeutic success, these UF probes improve upon current milk sampling techniques to more accurately determine PK data. For infections in the mammary gland, quantifying the concentrations of free drug in the gland cistern can help determine adequate concentrations of active drug in the udder. Plasma concentrations (Figure 3) were taken to demonstrate that only 1 dose of FLU was administered intravenously and plasma PK parameters (Table 1) were similar to previously reported studies (Kissell et al., 2012, 2015).

When milk is only sampled when cows are being milked, the total milk concentrations may not accurately reflect the exposure of the drug in the cistern (Stockler et al., 2009). Total milk samples collected every $12 \mathrm{~h}$ identified 1 maximum concentration of FLU

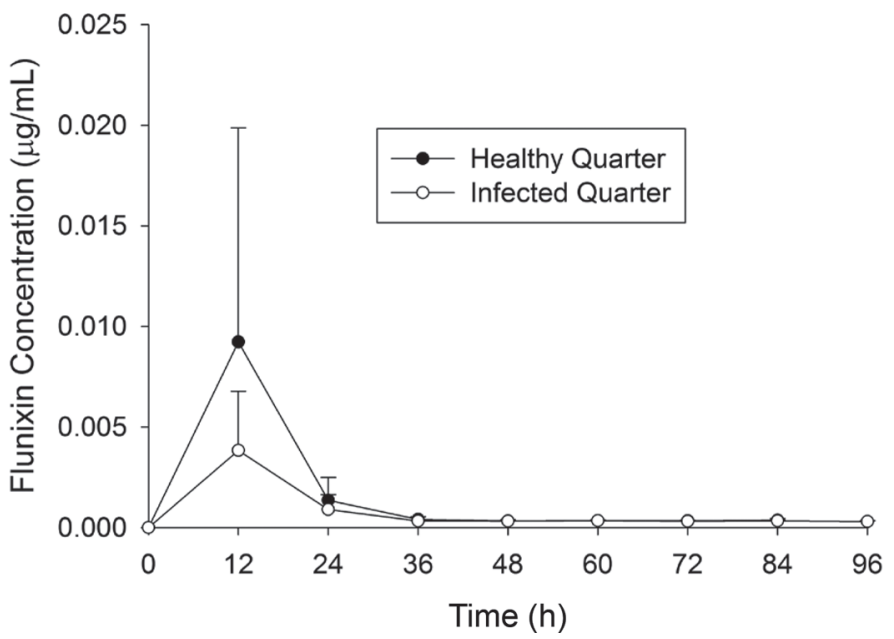

Figure 5. Total concentrations of flunixin in milk in healthy and mastitic quarters (mean $\pm \mathrm{SD}$ ) following single intravenous administration of $2.2 \mathrm{mg} / \mathrm{kg}$ of flunixin meglumine. 
at $12 \mathrm{~h}$, followed by a decline in concentration at each sampling point.

Whole milk concentrations of FLU peaked at $12 \mathrm{~h}$ (first sample after dosing). Mean concentrations of FLU at $12 \mathrm{~h}$ were higher in whole milk $(0.01 \pm 0.01$ $\mu \mathrm{g} / \mathrm{mL})$ than in milk ultrafiltrate $(0.005 \pm 0.002 \mu \mathrm{g} /$ $\mathrm{mL}$ ) in healthy quarters. In mastitic quarters, the geometric mean FLU concentrations were similar in milk ultrafiltrate $(0.0039 \pm 0.005 \mu \mathrm{g} / \mathrm{mL})$ to mean values found in whole milk $(0.0038 \pm 0.003 \mu \mathrm{g} / \mathrm{mL})$ at 12 h. Interpretation of these differences between whole milk as compared with milk ultrafiltrate is difficult, as a small sample size is confounded by the variability between cows. Although not evaluated in our study, the binding of FLU to milk proteins may also contribute to the wide variability in concentrations. Ultrafiltrate samples represent fluid collection over a period of time (i.e., not an instantaneous sample); this indicates that the concentration in the ultrafiltrate represents free drug collected over a set amount of time. At the 12-h sample, milk ultrafiltrate was collected for $4 \mathrm{~h}$. When corrected for an average concentration collected per hour, the concentration in milk ultrafiltrate at the 12-h sample in healthy and infected quarters was around 7 and $81 \%$ of the whole milk concentration, respectively. Flunixin is a highly protein-bound drug, with over $99 \%$ of the administered drug bound to plasma proteins in cattle (Odensvik and Johansson, 1995). Concentrations of FLU quantified in the milk ultrafiltrate represent free, unbound drug, whereas whole milk represents both bound and free drug. Future studies should evaluate binding of FLU to proteins present in milk and how disease within the mammary gland affects free concentrations of drugs.

Data collected from each cistern (healthy and mastitis quarters) through the UF probe identified 2 peak concentrations (Figure 4). The 2 peak concentrations we observed in FLU concentrations within each quarter may be the result of uneven distribution of drug in the mammary gland and dilution of drug when new milk is being produced. In mastitis, bacteria invade the mammary alveolar lumen and disrupt the blood-milk barrier. In a normal mammary gland, tight junctions maintain the blood-milk barrier of alveolar epithelium and block leakage of milk components into systemic circulation (Kobayashi et al., 2013). When this barrier is disrupted by pathogenic bacteria within the mammary gland, blood constituents can leak into the lumen of the alveolus, disrupt blood flow, decrease milk production, and change the protein profile of the milk, all of which may affect the distribution of drugs within the cistern and udder. The 2 peak concentrations that occurred at $12 \mathrm{~h}$ and 28 to $32 \mathrm{~h}$ after the single dose of FLU may reflect a lower volume of milk produced (decreased dilution) as well as changes in the mammary tissue within the cistern the probe is collecting from. Mastitis effects on milk yield are determined by a causative agent and on the degree it colonizes the mammary gland (Botaro et al., 2015). Edema or inflammation of the gland and blockage of ducts by necrotic or inflammatory debris can also affect distribution of drugs (Mestorino and Jorge, 2012). For infections in the mammary gland, data collected from these UF probes can help determine adequate concentrations of free drug in the udder and improve future PK studies of the mammary gland.

Failure to recognize the difference between total and unbound drug concentrations can lead to errors in the interpretation of the dose-exposure-response relationships. Whole milk samples represent total drug concentrations, bound and unbound, in milk proteins. The milk ultrafiltrate represent unbound drug within the cistern. The evaluation of the unbound and total drug concentration relationships within the cistern can improve predictions of possible changes in PK as well as safety or effectiveness for new drug approvals for mastitis treatment. Future examination of milk protein-drug interactions should be evaluated.

This method of sampling can provide a better understanding of how drugs partition into milk as well as factors that influence the distribution of antibiotics into the mammary gland. As cows can be continually milked on a normal schedule, the effects of milk production and milking frequency on drug distribution and elimination can be measured. This information would greatly improve bioequivalence studies when evaluating intramammary formulations of antimicrobials and other drugs.

The small number of cows, and therefore small number of sampled quarters, enrolled in our study represent limitations in data interpretation. Further, numerous pathophysiological factors associated with mastitis suggest that these findings may not be applicable to other infectious agents. Nonetheless, the use of UF probes allowed the identification of changes in drug concentration within the cistern that are missed if samples are only taken at each milking. Duration of sample collection is unknown, but our experience in other studies suggest that collection for up to a week is possible.

In summary, collection of UF samples from the mammary gland of cows provides a viable means to continuously assess drug concentrations in the milk while continuing to milk the cow normally. This allows for optimal PK modeling of active drug concentrations over time. These studies demonstrate the utility of continuous sampling of milk via UF for future PK studies in cattle. 


\section{ACKNOWLEDGMENTS}

Funding for this project was supported by the Food Animal Residue Avoidance Databank (Raleigh, NC).

\section{REFERENCES}

Al-Bataineh, M. M., D. van der Merwe, B. D. Schultz, and R. Gehring. 2009. Cultured mammary epithelial monolayers (BME-UV) express functional organic anion and cation transporters. J. Vet. Pharmacol. Ther. 32:422-428.

Bidgood, T. L., and M. G. Papich. 2003. Comparison of plasma and interstitial fluid concentrations of doxycycline and meropenem following constant rate intravenous infusion in dogs. Am. J. Vet. Res. 64:1040-1046.

Botaro, B. G., C. S. Cortinhas, A. G. Dibbern, L. F. P. E. Silva, N. R. Benites, and M. V. dos Santos. 2015. Staphylococcus aureus intramammary infection affects milk yield and SCC of dairy cows. Trop. Anim. Health Prod. 47:61-66.

Cagnardi, P., C. Locatelli, C. Ferraresi, V. Bronzo, S. Carli, R. Villa, and A. Zonca. 2014. Pharmacokinetics in foremilk and antimicrobial activity of cephapirin following intramammary administration in healthy and Staphylococcus aureus-infected cows. N. Z. Vet. J. 62:146-151.

Davis, J. L., D. M. Foster, and M. G. Papich. 2007. Pharmacokinetics and tissue distribution of enrofloxacin and its active metabolite ciprofloxacin in calves. J. Vet. Pharmacol. Ther. 30:564-571.

Davis, J. L., J. H. Salmon, and M. G. Papich. 2006. Pharmacokinetics and tissue distribution of doxycycline after oral administration of single and multiple doses in horses. Am. J. Vet. Res. 67:310-316.

Ehinger, A. M., and M. Kietzmann. 2000. Tissue distribution of oxacillin and ampicillin in the isolated perfused bovine udder. J. Vet. Med. A Physiol. Pathol. Clin. Med. 47:157-168.

Kissell, L. W., T. L. Leavens, R. E. Baynes, J. E. Riviere, and G. W. Smith. 2015. Comparison of pharmacokinetics and milk elimination of flunixin in healthy cows and cows with mastitis. J. Am. Vet. Med. Assoc. 246:118-125.

Kissell, L. W., G. W. Smith, T. L. Leavens, R. E. Baynes, H. Wu, and J. E. Riviere. 2012. Plasma pharmacokinetics and milk residues of flunixin and 5-hydroxy flunixin following different routes of administration in dairy cattle. J. Dairy Sci. 95:7151-7157.
Kobayashi, K., S. Oyama, A. Numata, M. M. Rahman, and H. Kumura. 2013. Lipopolysaccharide disrupts the milk-blood barrier by modulating claudins in mammary alveolar tight junctions. PLoS One 8:e62187.

Lainesse, C., R. Gehring, K. Pasloske, G. Smith, S. Soback, S. Wagner, and T. Whittem. 2012. Challenges associated with the demonstration of bioequivalence of intramammary products in ruminants. J. Vet. Pharmacol. Ther. 35(Suppl 1):65-79.

Messenger, K. M., M. G. Papich, and A. T. Blikslager. 2012. Distribution of enrofloxacin and its active metabolite, using an in vivo ultrafiltration sampling technique after the injection of enrofloxacin to pigs. J. Vet. Pharmacol. Ther. 35:452-459.

Mestorino, N., and O. Jorge. 2012. Pharmacokinetic-pharmacodynamic considerations for bovine mastitis treatment. Pages 423-472 in A Bird's-Eye View of Veterinary Medicine. C.C. Perez-Marin, ed. InTech. Rijeka, Croatia.

Odensvik, K., and I. M. Johansson. 1995. High-performance liquid chromatography method for determination of flunixin in bovine plasma and pharmacokinetics after single and repeated doses of the drug. Am. J. Vet. Res. 56:489-495.

Sojka, J. E., S. B. Adams, C. Rohde, and E. M. Janie. 2000. Surgical implantation of ultrafiltration probes in ovine bone and muscle. J. Invest. Surg. 13:289-294.

Stebler, T., and T. W. Guentert. 1990. Binding of drugs in milk: The role of casein in milk protein binding. Pharm. Res. 7:633-637.

Stockler, R. M., D. E. Morin, R. K. Lantz, W. L. Hurley, and P. D. Constable. 2009. Effect of milk fraction on concentrations of cephapirin and desacetylcephapirin in bovine milk after intramammary infusion of cephapirin sodium. J. Vet. Pharmacol. Ther. 32:345-352.

Sundlof, S. F., J. B. Kaneene, and R. A. Miller. 1995. National survey on veterinarian-initiated drug use in lactating dairy cows. J. Am. Vet. Med. Assoc. 207:347-352.

Underwood, C., S. N. Collins, A. W. van Eps, R. E. Allavena, C. E. Medina-Torres, and C. C. Pollitt. 2014. Ultrafiltration of equine digital lamellar tissue. Vet. J. 202:314-322.

Warren, C. D., T. Prange, N. B. Campbell, M. P. Gerard, L. G. Martin, M. E. Jacob, G. W. Smith, M. G. Papich, and D. M. Foster. 2014. Implantation of an ultrafiltration device in the ileum and spiral colon of steers to continuously collect intestinal fluid. Res. Vet. Sci. 97:611-615. 